\title{
ARTICLE Specific activation of mGlu2 induced IGF-1R transactivation in vitro through FAK phosphorylation
}

\author{
Yong-jian $\mathrm{Hu}^{1}$, Qian Sun ${ }^{1}$, Wen-hua Zhang ${ }^{1}$, Yu-jia Huo ${ }^{1}$, Chan-juan $\mathrm{Xu}^{1}$ and Jian-feng Liu ${ }^{1}$
}

\begin{abstract}
Metabotropic glutamate receptor 2 ( $\mathrm{mGlu2}$ ) belongs to the group-II metabotropic glutamate ( $\mathrm{mGlu}$ ) receptors and is a neurotransmitter $\mathrm{G}$ protein-coupled receptor. The group-II mGlu receptors are promising antipsychotic targets, but the specific role of mGlu2 signaling remains unclear. Receptor tyrosine kinases (RTKs) are also believed to participate in brain pathogenesis. To investigate whether there is any communication between mGlu2 and RTKs, we generated a CHO-mGlu2 cell line that stably expresses mGlu2 and showed that activation of mGlu2 by LY379268, a group II mGlu agonist, was able to transactivate insulin-like growth factor 1 receptor (IGF-1R). We further determined that the $G_{i / o}$ protein, $G_{\beta \gamma}$ subunits, phospholipase $C$, and focal adhesion kinase (FAK) were involved in the IGF-1R transactivation signaling axis, which further induced the phosphorylation of extracellular signal-regulated kinase1/2 (ERK1/2) and CAMP response element-binding protein. In primary mouse cortical neurons, similar signaling pathways were observed when mGlu2 were stimulated by LY487379, an mGlu2 positive allosteric modulator. Transactivation of IGF-1R through FAK in response to mGlu2 should provide a better understanding of the association of mGlu2 with brain disease.
\end{abstract}

Keywords: metabotropic glutamate receptor 2; insulin-like growth factor 1 receptor; transactivation; FAK; ERK1/2; LY379268; LY487379; mouse cortical neurons

Acta Pharmacologica Sinica (2019) 40:460-467; https://doi.org/10.1038/s41401-018-0033-7

\section{INTRODUCTION}

Metabotropic glutamate receptor 2 (mGlu2) belongs to the metabotropic glutamate (mGlu) group II receptors. These receptors are neurotransmitter $\mathrm{G}$ protein-coupled receptors (GPCRs) that modulate glutamatergic neuronal activity [1]. mGlu2 is composed of an extracellular Venus Flytrap domain, a cysteinerich domain, and a heptahelical domain and is predominantly coupled to $G_{i / o}$ proteins, which leads to inhibition of cAMP formation and voltage-gated $\mathrm{Ca}^{2+}$ channels and activation of $\mathrm{K}^{+}$ channels. Extensive studies indicate that activation of group II mGlu receptors may have promising antipsychotic effects $[2,3]$. The drugs targeting to mGlu2 are expected to be the next generation of antipsychotics, which may significantly improve both positive and negative symptoms in patients with schizophrenia. These new antipsychotics are intriguing since the current antipsychotics that target the dopamine and serotonin pathways have more effects on positive symptoms [3, 4]. However, the failure of group II mGlu receptor agonists in Phase II clinical trials $[5,6]$ has been a hindrance to their advancement to market.

There is debate on the potential for agonists of group-II mGlu receptors to be used as therapeutic agents for the treatment of schizophrenia: "Still promising or a dead end?" [7]. Owing to the conservation of Venus Flytrap domain, group-II mGlu agonists are unable to discriminate between mGlu2 and mGlu3 [8]. Overcoming this problem will require clear elucidation of the mechanisms of activation associated with each receptor subtype. Studies suggest distinct and important roles for mGlu2. For example, mGlu2 is enriched in the medial prefrontal cortex, which is linked to schizophrenia, whereas the dorsolateral entorhinal cortex and piriform cortex regions are enriched with mGlu3 [9]. Moreover, results from mGlu2 and mGlu3 knockout mice suggest that mGlu2 plays an important role in mediating antipsychotic activity in schizophrenia $[10,11]$, while mGlu3 is crucial in exerting neuroprotective effects [12]. While more selective and potent mGlu2 agonists or positive allosteric modulators (PAMs) are currently being developed, the understanding of the precise mechanisms of mGlu2 signaling remains incomplete, and additional studies are necessary.

Receptor tyrosine kinases (RTKs) communicate with GPCR signaling. RTKs, such as epidermal growth factor, plateletderived growth factor, brain-derived neurotrophic factor, nerve growth factor, and insulin like growth factor 1 (IGF-1), can be transactivated in response to GPCR activation by neurotransmitters and neuropeptides, which elicits a wide variety of functions [13]. Activation of IGF-1 receptor (IGF-1R) increases extracellular signal-regulated kinase1/2 (ERK1/2) phosphorylation, which might be beneficial to neuronal survival, brain development, and cognitive function [14]. Moreover, IGF-1 has been identified as a promising target or marker in brain disorders, such as schizophrenia [15-17] and Alzheimer's disease [18, 19]. IGF-1R is

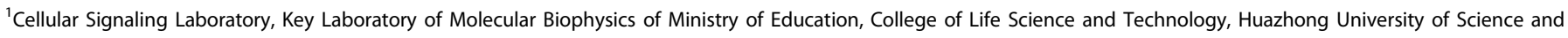
Technology, Wuhan 430074, China

Correspondence: Chan-juan Xu (chanjuanxu@mail.hust.edu.cn) or Jian-feng Liu (jfliu@mail.hust.edu.cn)

These authors contributed equally: Yong-jian Hu, Qian Sun.
}

Received: 26 January 2018 Accepted: 20 April 2018

Published online: 26 June 2018 
transactivated by angiotensin II type 1 receptor and $G_{A B A}$ receptor [20, 21]. However, whether there is crosstalk between mGlu2 and IGF-1R remains to be elucidated.

In the current study, we aimed to characterize the downstream signaling pathway of mGlu2. We found that mGlu2 activation resulted in transactivation of IGF-1R and further increased ERK1/2 and CAMP response element-binding protein (CREB) phosphorylation. It was determined that the $G_{i / o}$ protein, $G_{\beta \gamma}$ subunits, phospholipase $C(P L C)$, and focal adhesion kinase (FAK) were involved in the IGF-1R signal axis induced by mGlu2. In addition, the results from cortical neurons using an mGlu2-specific PAM confirmed that activation of IGF-1R by endogenous mGlu2 was similar to that in the heterogeneous expression system. IGF-1R and the downstream signaling axis activated in response to mGlu2 should provide a better understanding of how mGlu2 is associated with brain disease.

\section{MATERIALS AND METHODS}

Drugs

The drugs LY379268, LY341495, LY487379, PF573228, and U0126 were purchased from Tocris Bioscience (Shanghai, China). Pertussis toxin (PTX), AG1024, and U73122 were purchased from Merck Biosciences (Shanghai, China). F-12 medium and other solutions used for cell culture were purchased from Thermo Fisher Scientific (Shanghai, China). Fetal bovine serum (FBS) was purchased from Zhejiang Tianhang Biotechnology, China. Bovine serum albumin (BSA) was purchased from Biosharp, Shanghai, China.

\section{Plasmids}

The pcDNA3-c-myc-CD8- $\beta A R K$ plasmid, which includes the CD8antigen membrane receptor and a domain containing the $G$ protein $\beta \gamma$ subunit-binding site of GRK2, was a generous gift from Dr. Philippe Rondard (Institut de Genomique Fonctionnelle, Montpellier, France).

\section{Antibodies}

Primary antibodies for the detection of specific phosphorylated forms and non-phosphorylated forms of the various target antigens included rabbit anti-Phospho-p44/42 mitogen-activated protein kinase (MAPK) (pERK1/2) (phosphorylated at Thr202/ Tyr204) antibody (\#9101), rabbit anti-p44/42 MAPK (ERK1/2) antibody (\#9102), rabbit anti-Phospho-IGF-I receptor $\beta$ (Tyr1135/ 1136) (19H7) antibody (\#3024), rabbit anti-IGF-I receptor $\beta$ antibody (111A9) (\#3018), rabbit anti-Phospho-FAK (Tyr397) antibody (\#3283), rabbit anti-FAK antibody (\#3285), mouse antiPhospho-CREB (Ser133) (1B6) antibody (\#9196), and rabbit antiCREB antibody (\#9197). All of these antibodies were purchased from Cell Signaling Technology (Shanghai, China).

$\mathrm{CHO}-\mathrm{mGlu} 2$ cell line generation and culture

A stable cell line overexpressing mGlu2 was established using Chinese hamster ovary cells Flp-In-CHO. Human GRM2 CDNA (NM_000839.4) was subcloned into the mammalian expression vector pcDNA5/FRT using the restriction enzymes Nhel and BamHI. The plasmid pCDNA5/FRT-mGlu2 was transfected into $\mathrm{CHO}$ cells under hygromycin B (Roche, Shanghai, China) selection. Transfected CHO-mGlu2 colonies were subjected to immunoblotting to determine the level of mGlu2 expression. The cell lines were cultured in F-12 medium supplemented with $10 \%$ FBS, $0.2 \mathrm{M}$ glutamine, and $0.5 \%$ penicillin/streptomycin. Cell cultures were incubated at $37^{\circ} \mathrm{C}$ in a humidified atmosphere containing $5 \% \mathrm{CO}_{2}$. Flp-In-CHO cell and pcDNA5/FRT vector were purchased from Invitrogen (Shanghai, China).

shRNA transfection

The short hairpin RNA (shRNA) plasmid used in the IGF-1R RNAi knockdown experiments was described previously [21]. The forward DNA template was gatccccgaagatttcacagtcaacttcctgtcagattgactgtgaaatcttcggtttttg, and the reverse DNA template was aattcaaaaaccgaagatttcacagtcaatctgacaggaagttgactgtgaaatcttcggg. IGF-1R shRNA or shRNA vectors were transfected following the protocol of Lipofectamine 2000 (Invitrogen, Shanghai, China). Briefly, shRNA and Lipofectamine 2000 were diluted in Opti-MEM (Invitrogen, Shanghai, China) and then combined for $20 \mathrm{~min}$ at room temperature to allow for the formation of transfection complexes. Complexes were added to cells for $6-8 \mathrm{~h}$ at $37^{\circ} \mathrm{C}$. The medium was exchanged with fresh growth medium, and cells were grown for another $72 \mathrm{~h}$ in culture before being used for experiments.

\section{Western blotting analysis}

$\mathrm{CHO}$ cell lysates were sonicated, and the total protein concentrations were determined using Bradford reagent (Beyotime Biotechnology, Shanghai, China). Samples were adjusted to equal protein concentrations; mixed with $4 \times$ loading buffer containing $200 \mathrm{mM}$ Tris- $\mathrm{HCl} \mathrm{pH} 6.8,40 \%$ glycerol, $8 \%$ sodium dodecyl sulfate (SDS), $0.4 \%$ bromophenol blue, and $400 \mathrm{mM}$ dithiothreitol; and heated at $100{ }^{\circ} \mathrm{C}$ for $5 \mathrm{~min}$. Equal amounts of protein $(10-20 \mu \mathrm{g})$ were resolved by SDS-polyacrylamide gel electrophoresis on $10 \%$ gels. Proteins were transferred to nitrocellulose membranes (Millipore, Shanghai, China) and blocked for $2 \mathrm{~h}$ at room temperature (approximately $21^{\circ} \mathrm{C}$ ) with $5 \%$ nonfat dry milk buffer dissolved in TBS-T (TBS buffer with $0.1 \%$ Tween 20). The blots were incubated overnight at $4{ }^{\circ} \mathrm{C}$ with primary antibodies diluted in TBS-T containing BSA, followed by incubation with horseradish peroxidase-conjugated secondary antibodies (1:20,000; Cell Signaling Technology, Shanghai, China) for $2 \mathrm{~h}$. Immunoblots were visualized using enhanced chemiluminescence reagents (Thermo Fisher Scientific, Shanghai, China) and imaged on X-ray film (Kodak, Shanghai, China). The immunoreactive band density was measured by the $\mathrm{NIH}$ Image software, ImageJ. All bands were normalized to the percentage of the total protein levels or $\beta$-actin control values.

Primary cortical neuronal cultures

All experiments were specifically designed to minimize the number of animals used and were approved by the Animal Experimentation Ethics Committee of the School of Life Science and Technology, Huazhong University of Science and Technology. Primary cortical neuronal cultures were prepared from day-18 embryonic mice as previously described [22]. Pregnant Kunming mice were obtained from Hubei Provincial Center for Disease Control and Prevention. Following dissection in ice-cold phosphate-buffered saline (PBS), the cerebral cortices were dissociated with a trypsin solution; plated at a density of $1-2 \times 10^{6}$ cells/dish onto poly-D-lysine (Sigma-Aldrich, Shanghai, China) coated 35-mm culture dishes; and cultured in Neurobasal medium (Gibico, Shanghai, China) supplemented with $10 \%$ FBS (Sijiqing, Hangzhou, China), B27 supplement, $0.5 \mathrm{mM}$ GlutaMAX, $0.5 \%$ penicillin/ streptomycin, and $25 \mu \mathrm{M}$ L-glutamine. The medium was exchanged $24 \mathrm{~h}$ later for serum-free Neurobasal medium supplemented with B27 supplement, $0.5 \mathrm{mM}$ GlutaMAX, and $0.5 \%$ penicillin/streptomycin. Half of the serum-free medium was replaced every 3-4 days. Cell cultures were maintained at $37^{\circ} \mathrm{C}$ in a humidified atmosphere containing $5 \% \mathrm{CO}_{2}$.

\section{Drug treatments}

For ERK, IGF-1R, FAK, and CREB activation assays, cultures were washed once with $\mathrm{Ca}^{2+}$-free HEPES-buffered solution (HBS) containing $10 \mathrm{mM}$ HEPES pH 7.4, $140 \mathrm{mM} \mathrm{NaCl}, 4 \mathrm{mM} \mathrm{KCl}, 2 \mathrm{mM}$ $\mathrm{MgSO}_{4}$, and $1 \mathrm{mM} \mathrm{KH} \mathrm{PO}_{4}$ and starved for $2 \mathrm{~h}$ in $\mathrm{Ca}^{2+}$-free $\mathrm{HBS}$ buffer. For inhibitor experiments, cells were preincubated at $37^{\circ} \mathrm{C}$ with or without the inhibitor dissolved in HBS for the indicated time periods before drug stimulation. For PTX treatment, cells were pretreated or mock treated for $14-16 \mathrm{~h}$ with PTX 
( $200 \mathrm{ng} / \mathrm{mL}$ ). Cells were then stimulated at the indicated times by incubation with LY379268, LY487379, or an inhibitor prepared in fresh HBS. At the end of the treatment, the cells were quickly washed with ice-cold $\mathrm{Ca}^{2+}$-free PBS, $\mathrm{pH}$ 7.4. The cells were then lysed with $150 \mu \mathrm{L}$ of SDS lysis buffer and placed immediately on ice. The cell lysates were analyzed by Western blotting. Drugs were dissolved in HBS with or without dimethyl sulfoxide (DMSO). When DMSO was used with the drugs, the same concentration of DMSO was added to the controls.

\section{Statistical analysis}

Data are presented as the mean \pm SEM of at least three independent experiments. Statistical analysis was performed by one-way analysis of variance followed by Tukey's post hoc test. Values with $P<0.05$ were considered statistically significant.

\section{RESULTS}

Activation of mGlu2 increased ERK1/2 phosphorylation through the $G_{i / o}$ protein, $G_{\beta \gamma}$ subunits, and PLC

Several reports have shown that the group II mGlu agonist LY379268 induced ERK1/2 phosphorylation [23-26]. To investigate the role of mGlu2, we generated a cell line that stably expressed human mGlu2 in Flp-In-CHO cells. Expression of mGlu2 in $\mathrm{CHO}-$ mGlu2 cells was verified using an mGlu2-specific antibody and $\mathrm{Ca}^{2+}$ release assay. The established cell line showed stable mGlu2 expression and an effective response (Figure S1).

ERK1/2 phosphorylation was detected in the $\mathrm{CHO}-\mathrm{mGlu} 2$ cell line by LY379268 treatment. At $100 \mathrm{nM}$, LY379268 caused a rapid transient increase in ERK1/2 phosphorylation in CHO-mGlu2 with no alteration of the overall ERK1/2 expression levels (Fig. 1a). ERK1/2 phosphorylation peaked at $5 \mathrm{~min}$ and then gradually decreased back to the basal level. LY379268-induced ERK1/2 phosphorylation was dose dependent (Fig. 1b). The $\mathrm{EC}_{50}$ was approximately $12.1 \pm 4.2 \mathrm{nM}(n=4)$. ERK $1 / 2$ was fully phosphorylated with $100 \mathrm{nM}$ LY379268. For subsequent experiments, we chose to use $100 \mathrm{nM}$ LY379268 for $5 \mathrm{~min}$ of stimulation, unless otherwise indicated.

To confirm specificity, CHO-mGlu2 cells were pretreated with the mGlu2 and mGlu3 antagonist LY341495 and MEK inhibitor U0126 prior to LY379268 stimulation. Both LY341495 and U0126 decreased LY379268 induction of ERK1/2 phosphorylation (Figure S2).

We further investigated the signaling of mGlu2 toward ERK1/2 activation. Pretreatment of CHO-mGlu2 cells with $200 \mathrm{ng} / \mathrm{mL}$ PTX for 14-18 h blocked LY379268 induction of ERK1/2 phosphorylation (Fig. 1c). The CD8- $\beta$ ARK molecule consists of the transmembrane domain of CD8 as the membrane anchor and C-terminal region of GRK2 ( $\beta A R K$ ) and is characterized as a $G_{\beta \gamma}$ scavenger [27]. Overexpression of CD8- $\beta$ ARK also inhibited LY379268induced ERK1/2 phosphorylation (Fig. 1d). Pretreatment of cells a
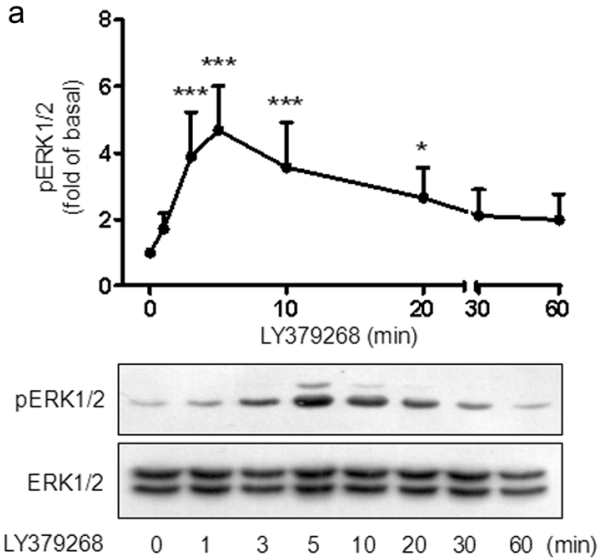

b
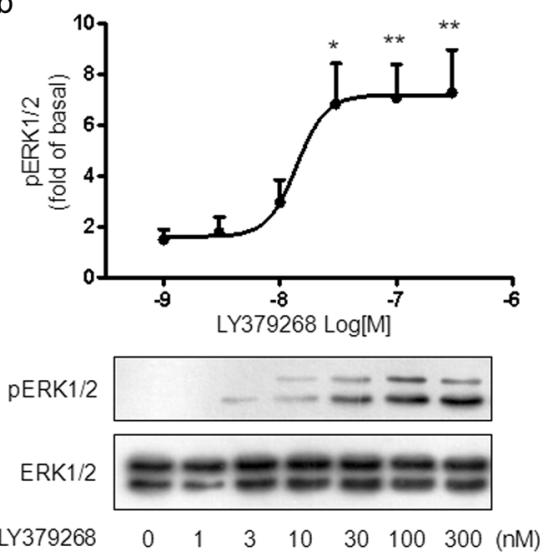

C

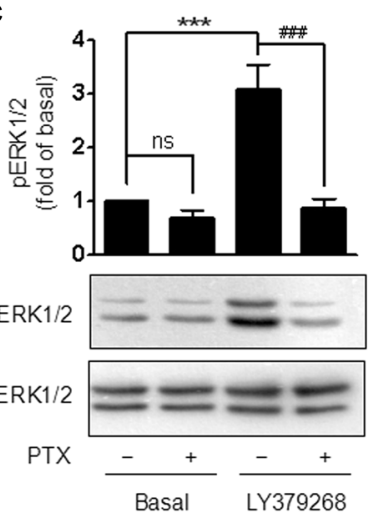

d
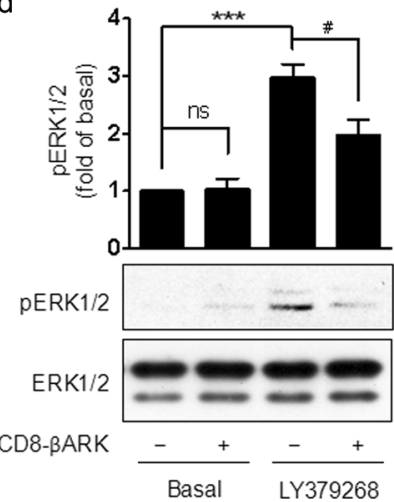

e

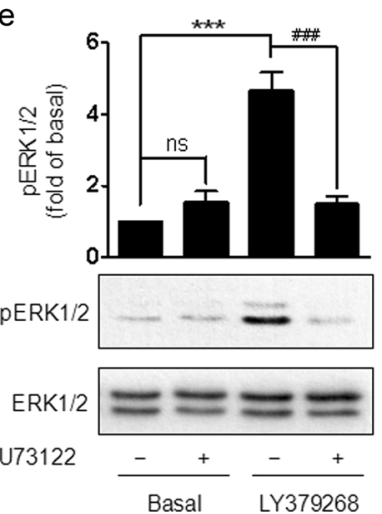

Fig. 1 Activation of mGlu2 increased ERK1/2 phosphorylation through $G_{i / o}, G_{\beta \gamma}$ and PLC in the CHO-mGlu2 cell line. a ERK1/2 phosphorylation following incubation with LY379268 (100 nM) for 0 to $60 \mathrm{~min}$. Phospho-ERK1/2 (pERK1/2) increased at 3 min and peaked at $5 \mathrm{~min}$, followed by decreasing at $10 \mathrm{~min}, 20 \mathrm{~min}$, and $60 \mathrm{~min}$ to the basal level. ${ }^{*} P<0.05,{ }^{* * *} P<0.001 \mathrm{vs}$. 0 min; $n=9$. b Dose response for the 5 min treatment of LY379268 on pERK1/2 in CHO-mGlu2 cells. An increase was observed at $30 \mathrm{nM}$ and reached a plateau at $100 \mathrm{nM}$. ${ }^{*} P<0.05$, ${ }^{*} P<0.01$ vs. $0 \mathrm{nM} ; n=4$. c-e CHO-mGlu2 cells were pretreated with PTX ( $\left.200 \mathrm{ng} / \mathrm{mL}\right)$ for $14-18 \mathrm{~h}$ or U73122 $(5 \mu \mathrm{M})$ for $1 \mathrm{~h}$ or were transfected with CD8- $\beta$ ARK for $48 \mathrm{~h}$, while there was no significant change of pERK1/2 compared with the basal level. LY379268 (100 nM, 5 min) increased ERK1/2 phosphorylation ( ${ }^{* * *} P<0.001 ; n \geq 3$ per group), which was inhibited by PTX $\left({ }^{\# \#} P<0.001 ; n=5\right)$, CD8- $\beta$ ARK $\left({ }^{\#} P<0.05 ; n=3\right)$, or U73122 $(\# \#) 0.001 ; n=3)$ 
with the selective PLC inhibitor U73122 abolished LY379268induced ERK1/2 phosphorylation (Fig. 1e) as well.

IGF-1R transactivated by mGlu2

To confirm the role of IGF-1R in mGlu2-induced ERK phosphorylation, we first used AG1024, an inhibitor of IGF-1R. AG1024 abolished LY379268 stimulation of ERK1/2 phosphorylation (Fig. 2a). Activated pIGF-1R was used as a control to confirm the inhibitory effect of AG1024 on IGF-1R. In parallel, we used IGF-1R shRNA to knockdown the endogenous levels of IGF-1R. LY379268activated ERK phosphorylation was reduced following interference with the IGF-1R shRNA (Fig. 2b). LY379268 caused a rapid and transient increase of IGF-1R phosphorylation without altering the IGF-1R protein expression levels (Fig. 2c, d).

$\mathrm{G}_{\beta \gamma}$ subunits, PLC, and FAK participated in mGlu2-induced IGF-1R transactivation

We then examined the role of the $G_{\beta \gamma}$ subunits and PLC in mGlu2-induced IGF-1R transactivation. Overexpression of CD8-ßARK or pretreatment with U73122 inhibited mGlu2activated IGF-1R phosphorylation (Fig. 3a, b).

FAK was reported to activate and stabilize the IGF-1R signaling complex [28]. In our study, LY379268 increased FAK phosphorylation (Fig. 3d) and the FAK inhibitor PF573228 abolished mGlu2induced IGF-1R, ERK1/2, and FAK phosphorylation in CHO-mGlu2 cells (Fig. $3 \mathrm{C}$ ), indicating that FAK was involved in the observed mGlu2-induced IGF-1R transactivation.
IGF-1R and ERK1/2 are involved in mGlu2-induced CREB phosphorylation CREB, an important transcription factor that regulates neurodevelopment, synaptic plasticity, and neuroprotection, was reported to function downstream of phosphorylated ERK1/2 [29]. We tested the response of CREB following mGlu2-mediated activation of the ERK1/2 pathway. We found that LY379268 transiently activated CREB phosphorylation in the CHO-mGlu2 cell line (Fig. 4a). Additionally, pretreatment of cells with AG1024 or U0126 blocked LY379268-induced CREB phosphorylation (Fig. 4b, c).

Endogenous mGlu2 transactivate IGF-1R to induce ERK $1 / 2$ and CREB phosphorylation in primary cortical neurons

Owing to the conservation of the mGlu Venus Flytrap domain, LY379268 is a specific agonist of group II mGlu, but it cannot discriminate between mGlu2 and mGlu3 [8]. PAMs, which bind to the heptahelical domain, display better selectivity among each subtype. The drug LY487379 (N-(4-(2-methoxyphenoxy)-phenyl)$\mathrm{N}$-(2,2,2-trifluoroethylsulfonyl)-pyrid-3-ylmethyl-amine) is a selective PAM of mGlu2 and has little activity on mGlu3 [30]. Using LY487379, we detected endogenous mGlu2 signaling in neurons. LY487379 stimulation increased IGF-1R, ERK1/2, and CREB phosphorylation in primary cortical neuronal cultures (Fig. 5a). After inhibition of IGF-1R by AG1024, the LY487379-activated levels of IGF-1R, ERK1/2, and CREB phosphorylation were decreased (Fig. 5b). a
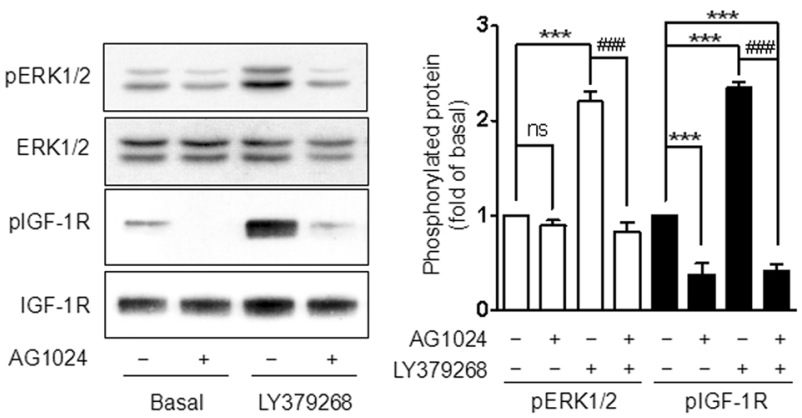

b

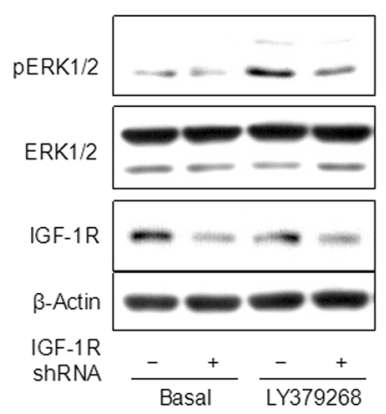

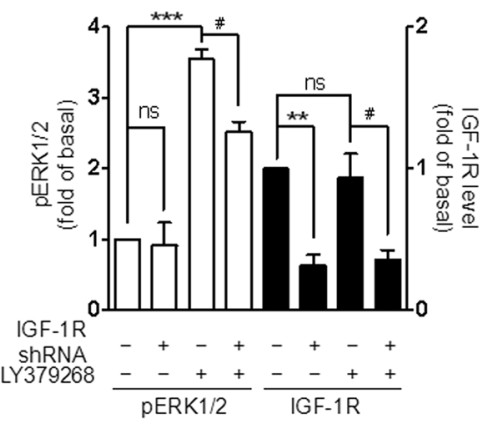

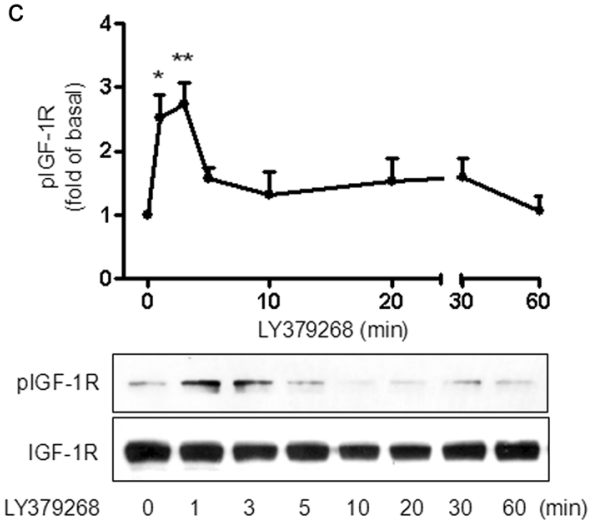

d

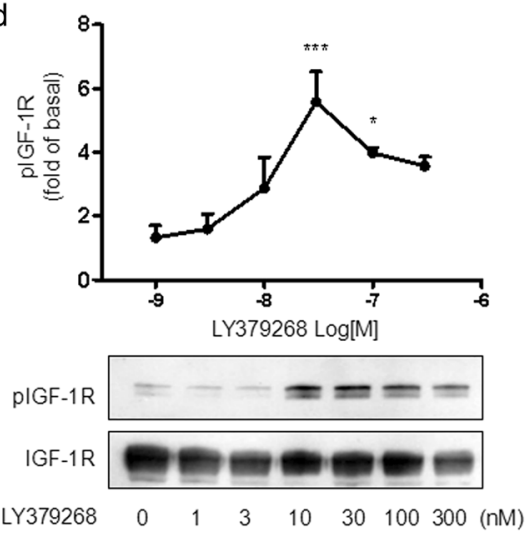

Fig. 2 Induction of ERK1/2 phosphorylation by mGlu2 through transactivation of IGF-1R. a Pretreatment with the IGF-1R inhibitor AG1024 $(0.1 \mu \mathrm{M}$ for $1 \mathrm{~h})$ did not change ERK1/2 phosphorylation in CHO-mGlu2 cells but inhibited the LY379268 (100 nM for $5 \mathrm{~min})$ increase of pERK1/

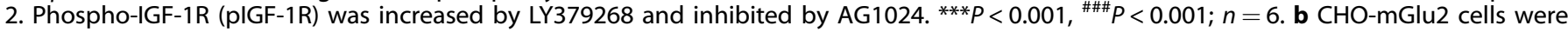
transfected with IGF1-R shRNA or control shRNA for $72 \mathrm{~h}$, followed by treatment with $100 \mathrm{nM}$ LY379268 for 5 min. IGF-1R shRNA decreased the expression of IGF-1R and decreased the LY379268 (100 nM for $5 \mathrm{~min}$ ) increase of pERK1/2. ${ }^{* *} P<0.01,{ }^{* * *} P<0.001,{ }^{*} P<0.05 ; n=3$. c Effect of LY379268 $(100 \mathrm{nM})$ treatment for different times on IGF-1R phosphorylation in CHO-mGlu2 cells. plGF-1R increased at 1 min to 3 min. ${ }^{*} P<$ 0.05 , ${ }^{* *} P<0.01$ vs. $0 \mathrm{~min} ; n=4$. d Effects of different concentrations of LY379268 on IGF-1R phosphorylation. At $30 \mathrm{nM}$ to $100 \mathrm{nM}$, LY379268 $(5 \mathrm{~min})$ treatment significantly increased pIGF-1R in CHO-mGlu2 cells. ${ }^{*} P<0.05,{ }^{* * *} P<0.001$ vs. $0 \mathrm{nM} ; n=3$ 
a

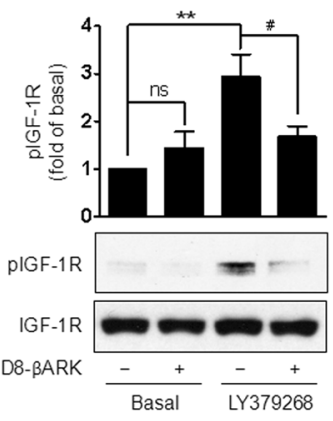

b

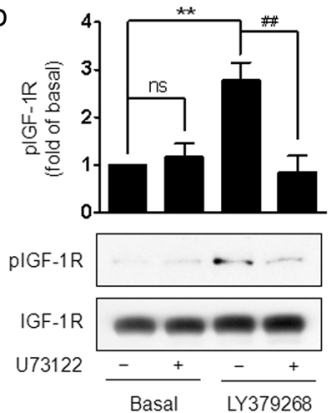

C

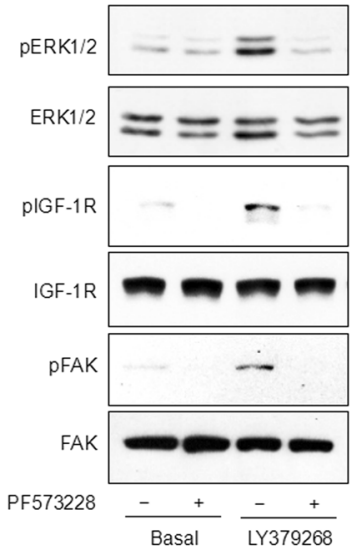

d
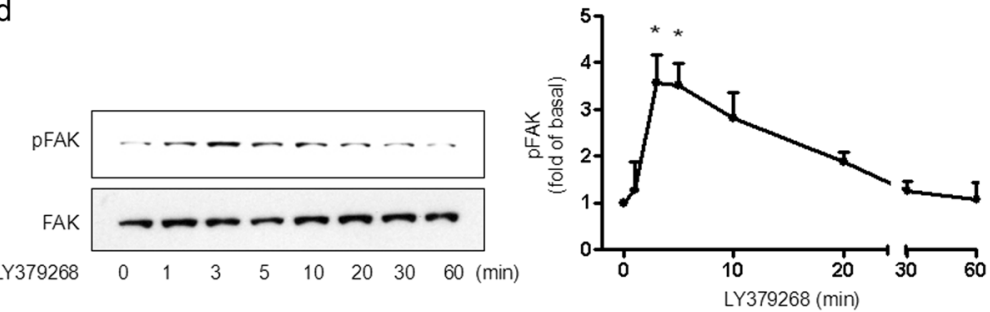

Fig. $3 \mathrm{G}_{\beta \gamma}$, PLC, and FAK are involved in mGlu2-induced IGF-1R phosphorylation. a The plasmid CD8- $\beta$ ARK was transfected into CHO-mGlu2 cells for $48 \mathrm{~h}$, followed by treatment with $100 \mathrm{nM}$ LY379268 for $5 \mathrm{~min}$. CD8- $\beta$ ARK did not change basal pERK1/2 but decreased LY379268induced pIGF-1R ( $\left.{ }^{* *} P<0.01,{ }^{*} P<0.05 ; n=5\right)$. b Pretreatment with U73122 (5 $\left.\mu \mathrm{M}, 60 \mathrm{~min}\right)$ inhibited LY379268 (100 nM, $\left.5 \mathrm{~min}\right)$-induced plGF-1R $\left({ }^{* *} P<0.01,{ }^{\# \# P} P<0.01 ; n=4\right)$. c Pretreatment of the FAK inhibitor PF573228 (10 $\left.\mu \mathrm{M}, 10 \mathrm{~min}\right)$ inhibited LY379268 (100 nM, $\left.5 \mathrm{~min}\right)$-induced pERK1/2 and pIGF-1R. Phospho-FAK (pFAK) was increased by LY379268 and inhibited by PF573228. ${ }^{* *} P<0.01,{ }^{* * *} P<0.001,{ }^{\# \# \#} P<0.001 ; n=4$. d FAK phosphorylation following incubation of CHO-mGlu2 cells with LY379268 (100 nM) for the time points indicated. pFAK increased after 3 min to 5 min incubation with LY379268. ${ }^{*} P<0.05$ vs. 0 min; $n=3$

a

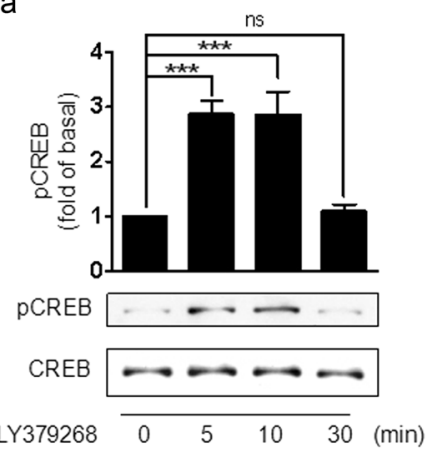

b
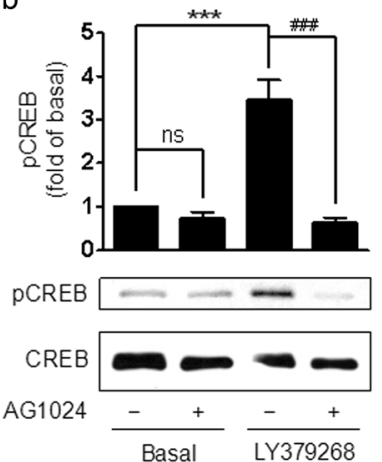
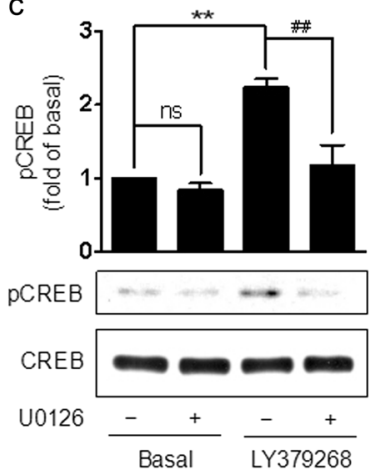

Fig. 4 mGlu2-induced CREB phosphorylation was dependent on the transactivation of IGF-1R and ERK1/2. a LY379268 (100 nM) treatment of CHO-mGlu2 cells increased CREB phosphorylation at $5 \mathrm{~min}$ to $10 \mathrm{~min}$, which decreased to the basal level after $30 \mathrm{~min}$. ${ }^{* * * P}<0.001 \mathrm{vs}$. 0 min; $n=5$. b-c LY379268 (100 nM, $5 \mathrm{~min}$ ) increased phospho-CREB (pCREB) was inhibited by pretreatment with the IGF-1R inhibitor, AG1024 $(0.1 \mu \mathrm{M}, 1 \mathrm{~h})\left({ }^{* *} P<0.001,{ }^{\# \# \#} P<0.001 ; n=5\right)$, and MEK inhibitor, U0126 $(10 \mu \mathrm{M}, 1 \mathrm{~h})\left({ }^{* *} P<0.01,{ }^{\# \#} P<0.01 ; n=6\right)$

\section{DISCUSSION}

RTKs can be transactivated by GPCRs, leading to signaling. There are an increasing number of studies pertaining to the crosstalk between GPCRs and RTKs [13], but this study is the first to show that mGlu2 is able to transactivate IGF-1R. Only a few GPCRs were reported to transactivate IGF-1R, such as $A T 1 R$ and $G_{A B A}$ receptor $[20,21]$. We identified the transactivation of IGF-1R by mGlu2 and the involvement of the $G_{\beta \gamma}$ subunits, PLC, and FAK in the activation process. Based on our findings and those of others, the non-RTK may be an important signal transducer. In AT1R transactivation of IGF-1R, Src, another non-RTK, was reported to be involved [20]. FAK was also found to act as a platform for protein-protein interactions and for the dissociation of $\mathrm{G}$ proteins, Src, and IGF-1R [31]. Meanwhile, our results demonstrated a role for the $G_{\beta \gamma}$ complex and PLC in the initiation of the mGlu2activated transduction, similar to the $\mathrm{GABA}_{B}$ receptor, which also belongs to the GPCR group $C$ and couples to the $G_{i / o}$ protein [21]. $\mathrm{PLC}$ is more often demonstrated to be activated by $\mathrm{G}_{\mathrm{q}}$-coupled 
a

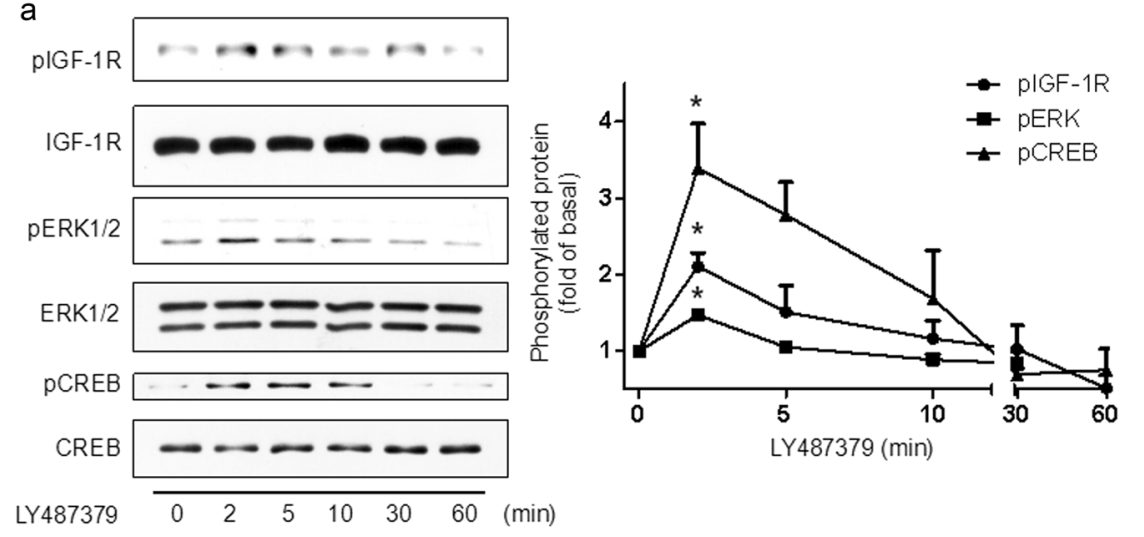

b
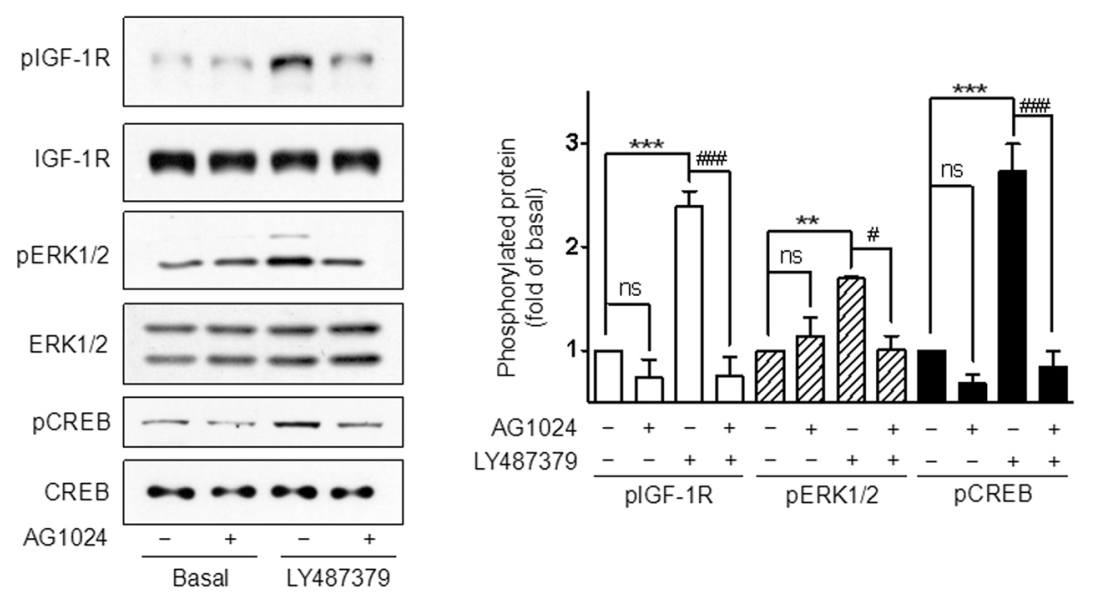

Fig. 5 mGlu2-specific PAM, LY487379, and transactivated IGF-1R are able to induce phosphorylation of ERK1/2 and CREB in cultured cortical neurons. a LY487379 $(1 \mu \mathrm{M})$ treatment transitorily increased the phosphorylation of IGF-1R $\left({ }^{*} P<0.05\right.$ vs. $\left.0 \mathrm{~min}, n=4\right)$, ERK1/2 $(* P<0.05$ vs. 0 min; $n=3$ ), and CREB ( ${ }^{P}<0.05$ vs. 0 min; $\left.n=3\right)$. b Effect of the IGF-1R inhibitor AG1024 $(0.1 \mu \mathrm{M}, 1 \mathrm{~h})$ on LY487379-induced ( $\left.1 \mu \mathrm{M}, 5 \mathrm{~min}\right)$ phosphorylation of ERK1/2 (pERK1/2) and CREB (pCREB). Phospho-IGF-1R (pIGF-1R) was increased by LY487379 and inhibited by AG1024. ${ }^{* *} P<0.01,{ }^{* * *} P<0.001,{ }^{\#} P<0.05,{ }^{\# \# \#} P<0.001 ; n=3$

GPCRs, but there are also several reports showing their involvement in $\mathrm{G}_{\mathrm{i} / \mathrm{o}}$-coupled GPCRs [21, 32], which is due to the activation of PLC by the $\mathrm{G}_{\beta \gamma}$ complex.

mGlu2 is a potential drug target for schizophrenia $[2,3]$. Decreased mGlu2 activity has been shown in schizophrenia animal models and patients [33-35]. The most reported mechanism of mGlu2 in schizophrenia is through an interaction and crosstalk with the $5 \mathrm{HT}_{2 \mathrm{a}}$ receptor. Decreased mGlu2 activity and hyperfunction of the $5 \mathrm{HT}_{2 \mathrm{a}}$ receptor is observed in schizophrenia [36], and agonists or PAMs of mGlu2 have been developed to correct this imbalance for treating schizophrenia. However, recently, mGlu2 activation was also found to activate $5 \mathrm{HT}_{2 \mathrm{a}}-\mathrm{G}_{\mathrm{q}}-\mathrm{Ca}^{2+}$ signaling directly [37]. The role of mGlu2 is not clearly elucidated in schizophrenia. The plasma levels of IGF-1 decrease in patients with schizophrenia, as well as their offspring [15, 17], while antipsychotic treatment induces a significant elevation of serum IGF-1 [16]. Here mGlu2transactivated IGF-1R indicated another role of mGlu2 leading to beneficial effects in schizophrenia by regulating IGF-1R signaling. Both $5 \mathrm{HT}_{2 \mathrm{a}}$ and IGF-1R contributed to mGlu2 downstream signaling via the $m G$ lu2-coupled $G_{i / o}$ and $G_{\beta \gamma}$ proteins [37]. Likewise, in an animal model of Alzheimer's disease, reduced IGF-1/IGF-1R signaling exhibited fewer amyloid plaques, less amyloid $\beta$, diminished neuroinflammation, improved spatial memory, and reduced anxiety and premature death $[18,19,38,39]$, while inhibition of mGlu2 activity with antagonists improved learning and reduced anxiety as well as the levels of brain amyloid $\beta$ in APP transgenic rodents [40]. Transactivation signaling from mGlu2 to IGF-1R supports the functional connections of the two receptors in pathological conditions.

Additionally, both mGlu2 and IGF-1R are widely expressed throughout the central nervous system. Activation of IGF-1R by mGlu2 leads to phosphorylation of ERK1/2/CREB, which plays important roles in long-term synaptic plasticity and in cognitive maintenance through regulation of protein synthesis and gene expression [29, 41]. CREB-targeted genes facilitate memory formation through induction of long-term synaptic plasticity, growth and formation of new synaptic spines and connections, and synthesis of new protein that participates in memory reconstruction [42]. Activation of IGF-1R to ERK and CREB phosphorylation should also contribute to the mGlu2 functions under normal physiological conditions.

There are reports showing the existence of mGlu2/4 heterodimers in transfected cell lines and native neurons, which can be activated by LY379268 $[43,44]$. However, little research has been reported on the distinct roles of the mGlu2 homodimer and mGlu2/4 heterodimer [45]. We used a stable cell line expressing mGlu2 to detect and evaluate the effects of mGlu2 alone. Additionally, LY487379, which is a specific PAM of mGlu2 and does not affect the mGlu2/4 heterodimer [44, 45], was used in primary cortical neurons. In both the stable mGlu2-expressing cell line and neurons, we detected similar transactivation of the IGF-1R signal axis by mGlu2. Further investigation on the mGlu2 


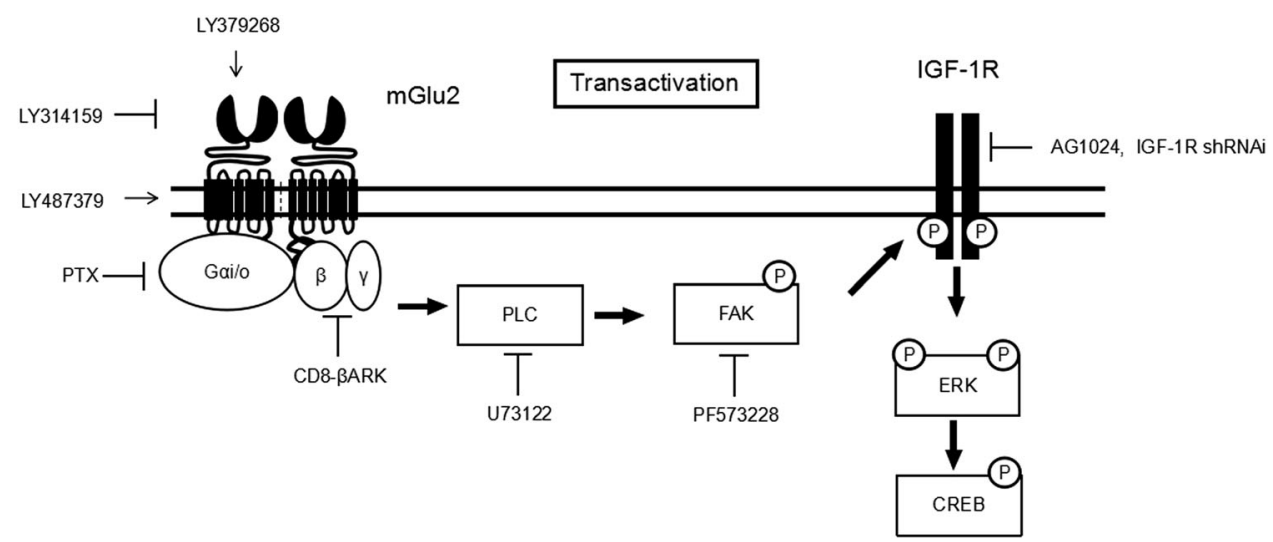

Fig. 6 Schematic representation of the mGlu2 signaling pathway. Activation of $m G l u 2$ by an agonist or PAM leads to the activation of $\mathrm{G}_{\mathrm{i} / \mathrm{o}}$ and $\mathrm{G}_{\beta \gamma}$ subunits, further activating PLC and FAK to transactivate IGF-1R, which induces ERK $1 / 2$ and CREB phosphorylation

homodimer will be important to better define the distinct roles of mGlu2.

In conclusion, for the first time, we identified the transactivation of IGF-1R by an mGlu2 agonist or PAM, which required $G_{i / o}, G_{\beta Y}$ subunits, PLC, and FAK, and led to the phosphorylation of ERK1/2 and CREB (Fig. 6). This signaling represents a new signaling mechanism of mGlu2 and suggests the role of the mGlu2 homodimer and its functional connection with IGF-1R in pathology.

\section{ACKNOWLEDGEMENTS}

The authors thank Jean-Philippe PIN and Philippe Rondard (Institut de Génomique Fonctionnelle, Montpellier, France) for their discussions and critical comments regarding the manuscript. This work was supported by the National Natural Science Foundation of China (NSFC grant nos. 31420103909, 31711530146, 31511130131), the Program of Introducing Talents of Discipline to the Universities of the Ministry of Education (grant no. B08029), Natural Science Foundation of Hubei Province (grant no. 2014CFA010), and NSFC grant (no. 31301163) to C-jX.

\section{AUTHOR CONTRIBUTIONS}

J-fL and C-jX conceived the project; Yong-jian Hu, QS, C-jX, and J-fL designed the experiments; Yong-jian Hu, QS, W-hZ, and Yu-jia Huo performed the experiments; Yong-jian $\mathrm{Hu}, \mathrm{QS}, \mathrm{C}-\mathrm{jX}$, and J-fL analyzed the data; and C-jX, J-fL, and Yong-jian Hu wrote the manuscript.

\section{ADDITIONAL INFORMATION}

The online version of this article (https://doi.org/10.1038/s41401-018-0033-7) contains supplementary material, which is available to authorized users.

Competing interests: The authors declare no competing interests.

Publisher's note: Springer Nature remains neutral with regard to jurisdictional claims in published maps and institutional affiliations.

\section{REFERENCES}

1. Nicoletti F, et al. Metabotropic glutamate receptors: from the workbench to the bedside. Neuropharmacology. 2011;60:1017-41.

2. Conn PJ, Lindsley CW, Jones CK. Activation of metabotropic glutamate receptors as a novel approach for the treatment of schizophrenia. Trends Pharmacol Sci. 2009;30:25-31.

3. Weinberger DR. Schizophrenia drug says goodbye to dopamine. Nat Med. 2007;13:1018-9.

4. Ellaithy A, Younkin J, Gonzalez-Maeso J, Logothetis DE. Positive allosteric modulators of metabotropic glutamate 2 receptors in schizophrenia treatment. Trends Neurosci. 2015;38:506-16.

5. Downing $\mathrm{AM}$, et al. A double-blind, placebo-controlled comparator study of LY2140023 monohydrate in patients with schizophrenia. Bmc Psychiatry. 2014;14:351.
6. Stauffer $V L$, et al. Pomaglumetad methionil: no significant difference as an adjunctive treatment for patients with prominent negative symptoms of schizophrenia compared to placebo. Schizophr Res. 2013;150:434-41.

7. Li ML, Hu XQ, Li F, Gao WJ. Perspectives on the mGluR2/3 agonists as a therapeutic target for schizophrenia: Still promising or a dead end? Prog Neuropsychopharmacol Biol Psychiatry. 2015;60:66-76.

8. Collado I, et al. $\left.2 S, 1^{\prime} S, 2^{\prime} S, 3^{\prime} R\right)-2-\left(2^{\prime}\right.$-carboxy-3'-methylcyclopropyl) glycine is a potent and selective metabotropic group 2 receptor agonist with anxiolytic properties. J Med Chem. 2002;45:3619-29.

9. Wright RA, et al. CNS distribution of metabotropic glutamate 2 and 3 receptors: transgenic mice and $[(3) \mathrm{H}] \mathrm{LY} 459477$ autoradiography. Neuropharmacology. 2013;66:89-98.

10. Fell MJ, Svensson KA, Johnson BG, Schoepp DD. Evidence for the role of metabotropic glutamate (mGlu) 2 not mGlu3 receptors in the preclinical antipsychotic pharmacology of the mGlu2/3 receptor agonist (-)-(1R,4S,5S,6S)-4-amino-2-sulfonylbicyclo[3.1.0] hexane-4,6-dicarboxylic acid (LY404039). J Pharmacol Exp Ther. 2008;326:209-17.

11. Woolley ML, Pemberton DJ, Bate S, Corti C, Jones DN. The mGlu2 but not the mGlu3 receptor mediates the actions of the mGluR2/3 agonist, LY379268, in mouse models predictive of antipsychotic activity. Psychopharmacol (Berl). 2008;196:431-40.

12. Durand D, Carniglia L, Caruso C, Lasaga M. mGlu3 receptor and astrocytes: partners in neuroprotection. Neuropharmacology. 2013;66:1-11.

13. Shah BH, Catt KJ. GPCR-mediated transactivation of RTKs in the CNS: mechanisms and consequences. Trends Neurosci. 2004;27:48-53.

14. Cheng $C M$, et al. Insulin-like growth factor 1 regulates developing brain glucose metabolism. Proc Natl Acad Sci USA. 2000;97:10236-41.

15. Venkatasubramanian $G$, et al. Insulin and insulin-like growth factor-1 abnormalities in antipsychotic-naive schizophrenia. Am J Psychiatry. 2007;164:1557-60.

16. Venkatasubramanian G, Chittiprol S, Neelakantachar N, Shetty T, Gangadhar BN. Effect of antipsychotic treatment on Insulin-like Growth Factor-1 and cortisol in schizophrenia: a longitudinal study. Schizophr Res. 2010;119:131-7.

17. Yang J, et al. Abnormality of glycometabolism related factors in non-psychotic offspring of schizophrenic patients. Psychiatry Res. 2012;198:183-6.

18. Freude $S$, et al. Neuronal IGF-1 resistance reduces Abeta accumulation and protects against premature death in a model of Alzheimer's disease. FASEB J. 2009;23:3315-24.

19. Gontier G, et al. Signaling in adult neurons alleviates alzheimer's disease pathology through amyloid-beta clearance. J Neurosci. 2015;35:11500-13.

20. Zahradka P, Litchie B, Storie B, Helwer G. Transactivation of the insulin-like growth factor-I receptor by angiotensin II mediates downstream signaling from the angiotensin II type 1 receptor to phosphatidylinositol 3-kinase. Endocrinology. 2004;145:2978-87.

21. Tu $\mathrm{H}$, et al. GABAB receptor activation protects neurons from apoptosis via IGF-1 receptor transactivation. J Neurosci. 2010;30:749-59.

22. Hilgenberg LG, Smith MA. Preparation of dissociated mouse cortical neuron cultures. J Vis Exp. 2007:562.

23. Phillips $T$, Barnes A, Scott $S$, Emson P, Rees $S$. Human metabotropic glutamate receptor 2 couples to the MAP kinase cascade in chinese hamster ovary cells. Neuroreport. 1998;9:2335-9.

24. Ferraguti F, Baldani-Guerra B, Corsi M, Nakanishi S, Corti C. Activation of the extracellular signal-regulated kinase 2 by metabotropic glutamate receptors. Eur J Neurosci. 1999;11:2073-82. 
25. Lin $\mathrm{CH}$, You JR, Wei KC, Gean PW. Stimulating ERK/PI3K/NFkappaB signaling pathways upon activation of mGluR2/3 restores OGD-induced impairment in glutamate clearance in astrocytes. Eur J Neurosci. 2014;39:83-96.

26. Saitoh F, Wakatsuki S, Tokunaga S, Fujieda H, Araki T. Glutamate signals through mGluR2 to control Schwann cell differentiation and proliferation. Sci Rep. 2016;6: 29856.

27. $\mathrm{Tu} H$, et al. Dominant role of GABAB2 and Gbetagamma for GABAB receptormediated-ERK1/2/CREB pathway in cerebellar neurons. Cell Signal. 2007;19: 1996-2002.

28. Andersson S, D'Arcy P, Larsson O, Sehat B. Focal adhesion kinase (FAK) activates and stabilizes IGF-1 receptor. Biochem Biophys Res Commun. 2009;387:36-41.

29. Johannessen M, Delghandi MP, Moens U. What turns CREB on? Cell Signal. 2004;16:1211-27.

30. Schaffhauser $\mathrm{H}$, et al. Pharmacological characterization and identification of amino acids involved in the positive modulation of metabotropic glutamate receptor subtype 2. Mol Pharmacol. 2003;64:798-810.

31. Lin $X$, et al. An activity-based probe reveals dynamic protein-protein interactions mediating IGF-1R transactivation by the GABA(B) receptor. Biochem J. 2012;443: 627-34.

32. Rozengurt E. Mitogenic signaling pathways induced by $G$ protein-coupled receptors. J Cell Physiol. 2007;213:589-602.

33. Kurita $M$, et al. HDAC2 regulates atypical antipsychotic responses through the modulation of mGlu2 promoter activity. Nat Neurosci. 2012;15:1245-54.

34. Matrisciano F, Tueting P, Maccari S, Nicoletti F, Guidotti A. Pharmacological activation of group-II metabotropic glutamate receptors corrects a schizophrenialike phenotype induced by prenatal stress in mice. Neuropsychopharmacology. 2012;37:929-38.
35. Gonzalez-Maeso J, et al. Identification of a serotonin/glutamate receptor complex implicated in psychosis. Nature. 2008;452:93-7.

36. Fribourg $M$, et al. Decoding the signaling of a GPCR heteromeric complex reveals a unifying mechanism of action of antipsychotic drugs. Cell. 2011;147: 1011-23.

37. Moreno JL, et al. Allosteric signaling through an mGlu2 and 5-HT2A heteromeric receptor complex and its potential contribution to schizophrenia. Sci Signal. 2016;9:ra5.

38. Cohen E, Bieschke J, Perciavalle RM, Kelly JW, Dillin A. Opposing activities protect against age-onset proteotoxicity. Science. 2006;313:1604-10.

39. Cohen $E$, et al. Reduced IGF-1 signaling delays age-associated proteotoxicity in mice. Cell. 2009;139:1157-69.

40. Kim SH, et al. Proneurogenic Group II mGluR antagonist improves learning and reduces anxiety in Alzheimer Abeta oligomer mouse. Mol Psychiatry. 2014;19: 1235-42.

41. Sweatt JD. The neuronal MAP kinase cascade: a biochemical signal integration system subserving synaptic plasticity and memory. J Neurochem. 2001;76: $1-10$.

42. Benito E, Barco A. CREB's control of intrinsic and synaptic plasticity: implications for CREB-dependent memory models. Trends Neurosci. 2010;33:230-40.

43. Yin $S$, et al. Selective actions of novel allosteric modulators reveal functional heteromers of metabotropic glutamate receptors in the CNS. J Neurosci. 2014;34: 79-94.

44. Moreno Delgado D, et al. Pharmacological evidence for a metabotropic glutamate receptor heterodimer in neuronal cells. eLife. 2017;6:e25233.

45. Liu J, et al. Allosteric control of an asymmetric transduction in a G proteincoupled receptor heterodimer. eLife. 2017;6:e26985. 